\title{
Chloroquine and Hydroxychloroquine and Covid-19: a systematic review of literature
}

José Paulo de Siqueira Guida'

Marcos Vinicius de Sousa ${ }^{2}$

Tabata Regina Zumpano dos Santos ${ }^{1}$

\section{ABSTRACT}

Objective: to assess effectiveness and safety of hydroxychloroquine and chloroquine on the treatment of COVID-19. Study desing: a systematic review of literature was performed in two databases; studies were included if they had as intervention use of chloroquine or hydroxychloroquine and reported outcomes on laboratorial or clinical findings or description of side effects. Results: 38 studies were included; of those, only one fulfilled inclusion criteria and were included in this review. This study has important methodological issues and only reported viral load, but any clinical outcomes. Conclusions: Many ongoing clinical trials will provide new evidences about the use of hydroxychloroquine and chloroquine to the treatment of COVID-19. Current evidence do not support its use on clinical practice.

Indexing terms: COVID-19; hydroxychloroquine; chloroquine

\section{INTRODUCTION}

The respiratory syndrome associated with the infection of SARS-Cov-2 was firstly reported in China and is now a public health concern, due to the fast spread of infection worldwide in the last months. The World Health Organization (WHO) declared it a pandemic, and global efforts are made to stop virus spread and to discover a drug to prevent complications and death associated to the disease, now recognized as COVID-19 (1).

Chloroquine, an anti-malarial drug, has been tested against other viral infections as MERS and SARS, with inconclusive results (2). With some known effects in in vitro testing, the same efficacy was not reported in clinical observations. However, due to the lack of other safe, proven and specific therapies, use of chloroquine against COVID-19 has been proposed (3).

The aim of this systematic review is to summarize results of previous studies that evaluated the use of chloroquine of hydroxychloroquine in patients with COVID-19, and to describe potential benefits, risks and efficacy of this therapy.

\section{METHODS}

We performed a systematic review of literature considering the following databases: MedLine (PubMed) and Scielo; we also assessed the Brazilian Registry of Clinical Trials (REBEC) and Clinicaltrials.gov to assess ongoing clinical trials in Brazil. We assessed those databases from Jan 012019 until Mar 30 2020, because description of first COVID-19 cases was on December 2019. There was no language restrictions.

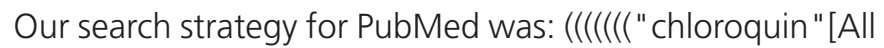
Fields]OR " chloroquine " [MeSHTerms])OR "chloroquine " [All Fields]) OR "chloroquines " [All Fields]) OR "chloroquines " [All Fields]) OR ("hydroxychloroquine"[MeSH Terms] OR " hydroxychloroquine" [All Fields])) OR "chloroquine "[MeSH Terms]) OR "hydroxychloroquine"[MeSH Terms]) AND

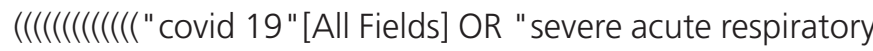
syndrome coronavirus 2"[Supplementary Concept]) OR "severe acute respiratory syndrome coronavirus 2"[All Fields]) OR "2019 ncov"[All Fields]) OR "sars cov 2"[All Fields]) OR "2019ncov" [All Fields]) OR (("wuhan "[All Fields] AND ("coronavirus"[MeSH Terms] OR "coronavirus"[All

\footnotetext{
${ }^{1}$ São Leopoldo Mandic School of Medicine. Rua José Rocha Junqueira, 13, Swift, 13045-755, Campinas, SP, Brazil. Corresponding to: JPS GUIDA. E-mail: <jose.guida@slmandic.edu.br>.

${ }^{2}$ Renal Transplant Unit, University of Campinas, Brazil.

ه曰田

Como citar este artigo / How to cite this article

Guida JPS, Sousa MV, Santos TRZ. Chloroquine and Hydroxychloroquine and Covid-19: a systematic review of literature. InterAm J Med Health 2020;3:e202003018
} 
Fields])) AND 2019/12/1:2030/12/31[Date - Publication])) OR (("covid 19"[Supplementary Concept] OR "covid 19"[All Fields]) OR "covid19"[All Fields])) OR ("severe acute respiratory syndrome coronavirus 2" [Supplementary Concept]OR "severe acute respiratory syndrome coronavirus 2 "[All Fields])) OR (("severe acute respiratory syndrome coronavirus 2" [Supplementary Concept] OR "severe acute respiratory syndrome coronavirus 2" [All Fields]) OR "2019 ncov"[All Fields])) OR (("coronavirus"[MeSH Terms] OR "coronavirus"[All Fields]) OR "coronaviruses"[All Fields])) OR "coronavirus"[MeSH Terms]) OR "pneumonia"[MeSH Terms]) OR (((("pneumonia"[MeSH Terms] OR "pneumonia"[All Fields]) OR "pneumoniae"[All Fields]) OR "pneumonias"[All Fields]) OR "pneumoniae s"[All Fields])). The search strategy for Scielo was ((COVID19)) OR (sars-cov-2) AND ((hydroxychloroquine) OR (chloroquine)). For REBEC, we searched "chloroquine", "cloroquina", "hydroxychloroquine" and "hidroxicloroquina"; and for clinicaltrials.gov we defined intervention as "chloroquine" and searched for studies ongoing in Brazil.

The two authors independently screened databases and selected relevant articles; data was extracted by the two authors and it was stored in RevMan 5.3. Inclusion criteria was results from trials or observational studies that evaluated use of chloroquine or hydroxychloroquine (isolated or in addition to other drugs) in clinical observations; we excluded letter to editor, in vitro studies, other systematic reviews and society recommendations. The outcomes considered were response to drug; occurrence of side effects; and laboratorial findings.

\section{RESULTS}

There are three clinical trials currently registered to be performed in Brazil. The number of participants, settings and proposed interventions are described in table 1 . Of those, one is already recruiting participants, while the other two are not in recruiting phase. In those trials, one will compare different doses of chloroquine, other will compare isolate hydroxychloroquine against its combination with azithromycin, and another comparing two different regimens of chloroquine and usual care to COVID-19.

Thirty-eight studies were found in PubMed, and no studies were found in Scielo. After title screening, 18 studies were opposed to inclusion and exclusion criteria: 17 studies were excluded 11 reviews, 3 letters and 2 consensus). One study was included (4).

In the included study, 36 patients were enrolled: 20 in the intervention group and 16 in the control group. The proposed intervention was chloroquine (with or without azithromycin) against regular care to Covid-19, and the outcome considered was viral load clearance in day 6. In experimental group, 14 patients had negative viral load at day $6(70 \%)$, against 2 in control group $(12.5 \%)$; others laboratorial or clinical outcomes were not reported.

About bias evaluation, the included study had high risk of bias on randomizing process, allocation concealment, blinding of participants and personnel and incomplete outcome data.

Tabela 1. Ongoing clinical trials testing chloroquine or hydroxychloroquine in Brazil

\begin{tabular}{c|c|c|c|c} 
Register & Center & $\begin{array}{c}\text { Number of } \\
\text { participants }\end{array}$ & Status \\
\hline $\begin{array}{c}\text { REBEC RBR-9d8z6m } \\
\text { and ClinicalTrials } \\
\text { NCT04322123 }\end{array}$ & $\begin{array}{c}\text { Sao Paulo and 32 other } \\
\text { study locations }\end{array}$ & 630 & $\begin{array}{c}\text { Three arms: usual care to COVID19 } \\
\text { versus azithromycin and chloroquine } \\
\text { versus chloroquine }\end{array}$ & Not yet recruiting \\
\hline $\begin{array}{c}\text { ClinicalTrials } \\
\text { NCT04323527 }\end{array}$ & $\begin{array}{c}\text { Delphina Aziz Hospital; } \\
\text { Manaus }\end{array}$ & 440 & $\begin{array}{c}\text { Two arms: chloroquine 600mg vs } \\
\text { chloroquine 450mg }\end{array}$ & Recruiting \\
\hline $\begin{array}{c}\text { ClinicalTrials } \\
\text { NCT04321278 }\end{array}$ & $\begin{array}{c}\text { Salbert Eaulo, and 21 other } \\
\text { study locations }\end{array}$ & 440 & $\begin{array}{c}\text { Two arms: hydroxychloroquine vs } \\
\text { hydroxychloroquine + azithromycin. }\end{array}$ & Not yet recruiting
\end{tabular}


Tabela 2. Included studies in this systematic review, with a brief description of intervention, outcomes and results.

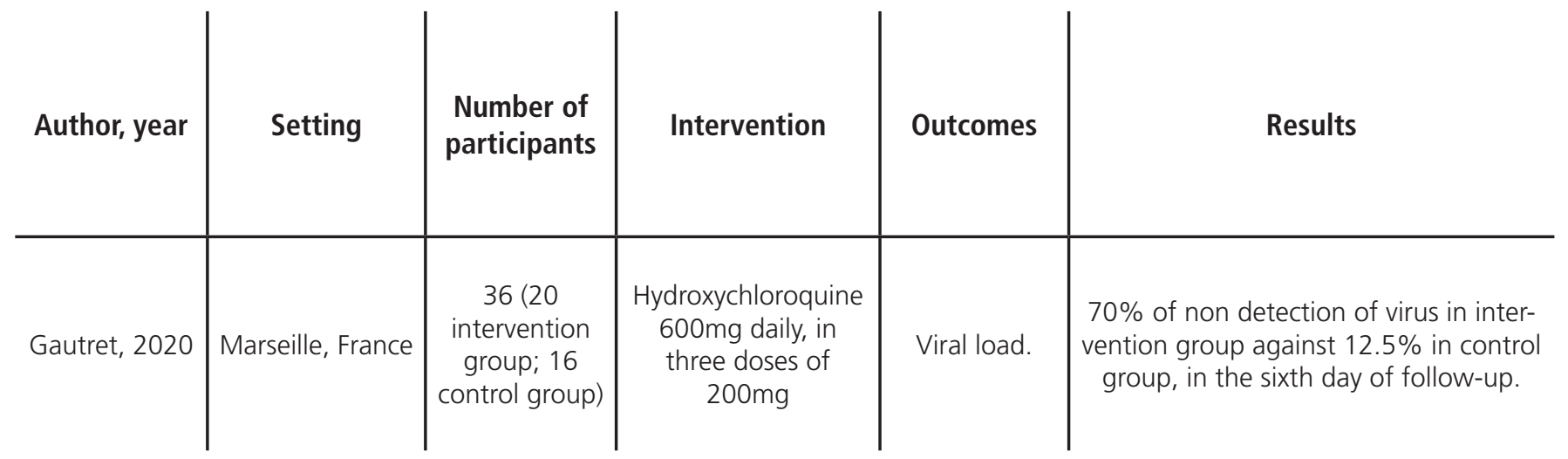

\section{DISCUSSION}

The current systematic review aimed to assess the use of chloroquine and hydroxychloroquine in the treatment of Covid-19, the most severe pandemic of this century and a current public heath issue.

Our results showed that only one study have been published describing use of hydroxychloroquine in patients with COVID-19. Majority of reports retrieved from our search in scientific databases were letters to editor, consensus based on specialists' opinions and reviews. Some of those report interesting results with use of chloroquine of hydroxychloroquine; however, objective results of the therapy are not available until now.

The only included study (4) has several concerns regarding some bias: selection and inclusion of patients in experimental group was not randomized. Patients were included in control group if they refused to undergo intervention or if they had any contraindication to use the drug. This is a clear selection bias. Researchers should exclude those patients of the study and randomize intervention among patients that fulfilled inclusion criteria.

Other bias is regarding incomplete outcome data: in intervention group, six patients were excluded (three due to admission in intensive care unit, one died, one left hospital and one stopped treatment). Results of these patients could change overall results of study and, of note that the only death occurred in intervention group.

Despite those methodological issues, there is one important problem regarding results: outcome chosen by researchers was clearance of viral load, instead of any clinical outcomes, as recovering from disease. By this time, we do not know if viral load is enough, or if the disease (or its consequences) can rest after clearance of viral in airways.
Our study has some limitations: only one study was included, and its results cannot be generalized. We only searched in two databases and it is possible that there are some studies available in other databases, however language barriers (Chinese, for example) could stop this review.

In the other hand, this review supports that, in this moment, there are not enough data to use chloroquine or hydroxychloroquine in clinical practice. There are many clinical trials ongoing evaluating use of this drug, and they will provide us more evidence about efficacy and safety of this drug against COVID-19.

As this is an emerging issue, and there are many clinical trials ongoing, we will update this review as new evidences are available.

\section{CONCLUSIONS}

Available evidence regarding use of hydroxychloroquine or chloroquine to treat COVID-19 do not support its use in clinical practice.

\section{REFERENCES}

1. World Health Organization. Coronavirus Disease 2019 (COVID-19) Situation Report 512020 [Available from: https://www.who.int/docs/defaultsource/coronaviruse/situation-reports/20200311sitrep-51-covid-19.pdf?sfvrsn=1 ba62e57_10.

2. Al-Bari MAA. Targeting endosomal acidification by chloroquine analogs as a promising strategy for the treatment of emerging viral diseases. Pharmacol Res Perspect. 2017;5(1):e00293. 
3. Colson P, Rolain JM, Raoult D. Chloroquine for the 2019 novel coronavirus SARS-CoV-2.

Int J Antimicrob Agents. 2020;55(3):105923.

4. Gautret P, Lagier JC, Parola P, Hoang VT, Meddeb L, Mailhe $M$, etal. Hydroxychloroquine and azithromycin as a treatment of COVID-19: results of an open-label non-randomized clinical trial. Int J Antimicrob Agents. 2020:105949. 